\title{
Jet hadrochemistry as a characteristics of jet quenching
}

\author{
Sebastian Sapeta ${ }^{1,2}$ and Urs Achim Wiedemann ${ }^{1}$ \\ ${ }^{1}$ Department of Physics, CERN, Theory Division, CH-1211 Geneva 23, Switzerland \\ ${ }^{2}$ M. Smoluchowski Institute of Physics, Jagellonian University, Reymonta 4, 30-059 Cracow, Poland
}

\begin{abstract}
Jets produced in nucleus-nucleus collisions at the LHC are expected to be strongly modified due to the interaction of the parton shower with the dense QCD matter. Here, we point out that jet quenching can leave signatures not only in the longitudinal and transverse jet energy and multiplicity distributions, but also in the hadrochemical composition of the jet fragments. In particular, we show that even in the absence of medium effects at or after hadronization, the medium-modification of the parton shower can result in significant changes in jet hadrochemistry. We discuss how jet hadrochemistry can be studied within the high-multiplicity environment of nucleus-nucleus collisions at the LHC.
\end{abstract}

\section{INTRODUCTION}

Highly energetic partons, propagating through dense QCD matter, are fragile. Their fragmentation pattern changes in the presence of a strongly interacting medium. This is reflected in the medium-modification of their hadronic remnants in nucleus-nucleus collisions at RHIC [1, 2, 3, 4 ] and will soon be further explored at the higher LHC collider energies [5, 6, 7, 8]. The generic suppression of high- $p_{T}$ single inclusive hadron spectra [9, 10, 11, 12, 13, 14, 15] and triggered particle correlations [16, 17, 18] in A-A collisions at RHIC support this picture. These measurements can be largely accounted for in models of radiative parton energy loss [19, 20, 21, 22, 23, 24], which assume that additional medium-induced parton splitting leads to a softening of the parton shower, and thus to an energy degradation of the leading hadrons. If the leading hadronic fragment in a jet carries less energy, then - due to energy-momentum conservation - either the subleading fragments carry more, or their multiplicity increases accordingly. The resulting changes expected for the longitudinal [25, 26, 27, 28] and transverse [25, 29, 30, 31, 32] jet energy and jet multiplicity distributions have been discussed in the context of models, which account successfully for the suppression of single inclusive hadron spectra. In this paper, we study a third class of jet characteristics, which may show significant medium effects: measurements of the hadrochemical composition of jet fragments.

There are several reasons of why one expects parton energy loss to affect jet hadrochemistry. In particular, in all models of radiative parton energy loss [33, 34, 35, 36, 37, 38], the interaction of a parent parton with the QCD medium transfers color between partonic projectile and target. This changes the color flow in the parton shower and is thus likely to affect hadronization. To illustrate this point, we have sketched in Fig. 1a an entirely gluonic parton shower in the large- $N_{c}$ approximation, where gluons are represented as $q \bar{q}$-dipoles. At large $N_{c}$, the color singlet prehadronic subsystems at the end of the perturbative evolution of the shower may be identified with the connected fermion lines in this diagram. Fig. 1b illustrates the conceivable effects of a single gluon exchange between the projectile gluon and a target quark in the medium. The multiplicity in the shower increases and the momentum distribution may soften and widen accordingly. Also, in general, the color singlet prehadronic subsystems at the end of the perturbative evolution will have a different distribution in invariant mass. This makes it likely that their subsequent fragmentation into hadrons results in a hadrochemical distribution different from that of a gluon fragmenting in the vacuum. This model-dependent example illustrates that existing models of parton energy loss, though formulated in an entirely partonic language, contain one possible ingredient for a mediuminduced modification of the hadrochemical composition of jets, namely: color exchange between projectile and target. 

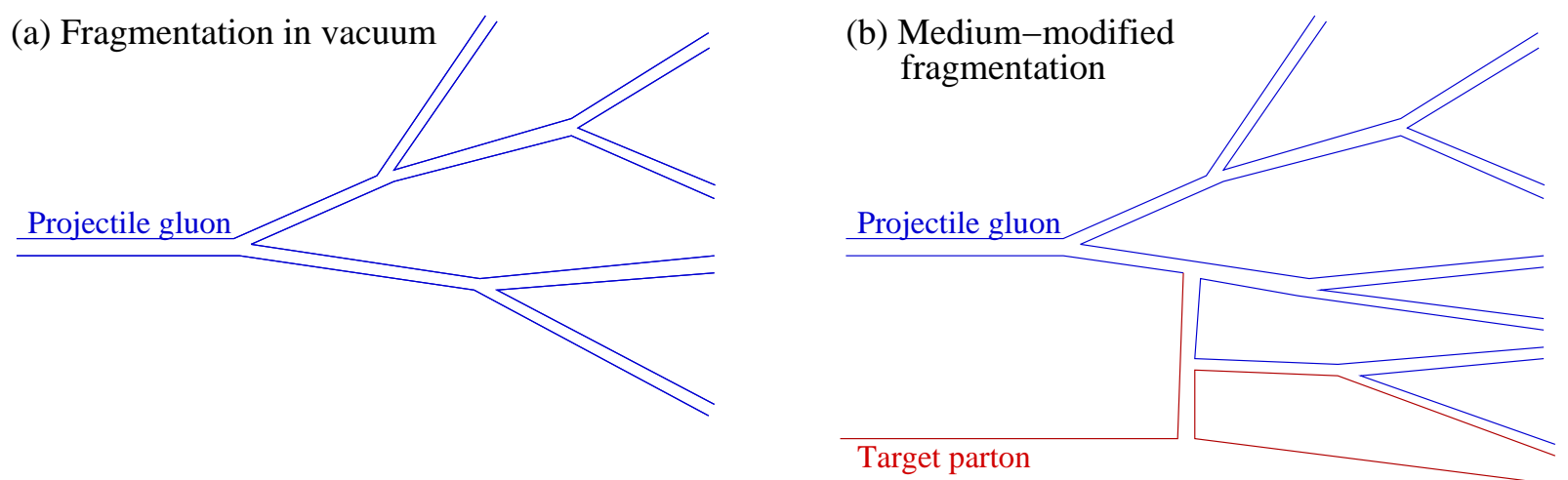

FIG. 1: Sketch of an entirely gluonic parton shower in the large $N_{c}$ limit, where gluons are represented as pairs of $q \bar{q}$ fermion lines, and quarks as single lines. (a) Fragmentation of the gluon in the vacuum. (b) Interaction of the gluon with a target quark in the medium via a single gluon exchange. This interaction changes the color flow and may affect hadronization, see text.

In addition, flavor or baryon number could be exchanged between medium and projectile. For instance, subleading partons in the jet may be subject to hadronization via recombination with partons from the medium [39, 40, 41, 42, 43, 44, 45], a mechanism which is conjectured to underlie baryon enhancement of identified single inclusive spectra at intermediate $p_{T}$. If parton energy loss involves non-negligible recoil effects (a.k.a. collisional energy loss [46, 47, 48]), then one may also speculate that the hard parton kicks components of the medium into the jet cone. Depending on the quantum numbers carried by the components kicked, this will affect jet hadrochemistry. In short, medium-induced modifications of jet hadrochemistry are a direct (for the case of color transfer) or conceivable (for the case of recombination, flavor and baryon number transfer) consequences of the current models of jet quenching. They provide complementary information about the microscopic mechanism underlying parton energy loss. Thus, jet hadrochemistry may be a valuable tool for establishing the properties of the matter produced in heavy ion collisions.

The interfacing of a parton shower with the hadronic world is a significant modeling task with very little guidance from theory, even for the simplest systems without medium-modifications, such as jets in $e^{+} e^{-}$collisions. This task is more complex for the case of a medium-modified jet, since it depends now additionally on information about the exchange of momentum, color, flavor and baryon number between the partonic projectile and the medium. In the present work, we shall largely bypass these complications. We merely observe that even if the exchange of quantum numbers between projectile and target is disregarded, a change of hadrochemical composition can be expected for the generic case that the distribution of partons in invariant mass is modified. It is a model of this type [27], which we introduce in section [I]. Since this model does not implement any of the effects mentioned above, it may be expected to underestimate the mediummodifications of jet hadrochemistry. Specific signatures of hadrochemical modifications, indicative e.g. of color, flavor or baryon number transfer, may be established as deviations from this model. In section III, we discuss the hadrochemical composition of the high-multiplicity soft background, in which medium-modified jets are immersed in heavy ion collisions. We emphasize that the hadrochemical composition of this background differs significantly from that of a proton-proton collision, and it also differs form the vacuum fragmentation of a jet. This difference in composition will help to characterize jet hadrochemistry in nucleus-nucleus collisions. Our main conclusions are summarized in section IV. 


\section{JET MULTIPLICITY DISTRIBUTIONS AND THEIR MEDIUM-MODIFICATION}

In this section, we introduce and study a specific model of how the single inclusive hadron distribution in a jet is composed of different hadron species, and how it changes due to mediumeffects.

\section{A. Single inclusive intrajet distributions in the absence of medium modification}

The single inclusive distribution $d N^{h} / d \xi$ of hadrons inside a jet, plotted versus the logarithm of the hadronic momentum fraction $\xi=\ln [1 / x], x=p_{h} / E_{\text {jet }}$, shows a characteristic hump-backed plateau. Measurements of this distribution in $e^{+} e^{-}$collisions and hadronic collisions are well described in the MLLA (modified leading logarithmic approximation) formalism [49, 50, 51, 52], supplemented by local parton-hadron duality (LPHD) [51, 53, 54]. Here, the MLLA is a perturbative calculation of the parton distribution $D_{q, g}(\xi, \tau, \lambda)$ inside a quark or gluon jet, which achieves double and single logarithmic accuracy in $\xi=\ln [1 / x]$ and $\tau=\ln [Q / \Lambda]$ where $\lambda=\ln \left[Q_{0} / \Lambda\right]$. It is based on leading order parton splitting functions and uses $Q \sim E_{\text {jet }}$ as the starting scale of the parton shower evolution. The evolution is stopped at the scale $Q_{0}$, which is, like $\Lambda$, a fit parameter of order $O\left(\Lambda_{\mathrm{QCD}}\right)$. The effects of destructive quantum interference which are responsible for the shape of the distribution translate at small $x$ into the prescription of angular ordering of a probabilistic parton cascade.

At high energies the spectrum turns out to be insensitive to the value of $Q_{0}$ and it is sufficient to consider the case $\lambda=0$, which is equivalent to $Q_{0}=\Lambda=Q_{\text {eff }}$. This is the so called limiting spectrum, which for gluon jets takes the form [49]

$$
\begin{aligned}
& D_{g}^{\lim }(\xi, Y)=A \Gamma(B) \int_{-\frac{\pi}{2}}^{\frac{\pi}{2}} \frac{d \tau}{\pi} e^{-B \alpha}\left[\frac{\cosh \alpha+(1-2 \zeta) \sinh \alpha}{A Y \frac{\alpha}{\sinh \alpha}}\right]^{B / 2} \\
& \times I_{B}\left(\sqrt{4 A Y \frac{\alpha}{\sinh \alpha}[\cosh \alpha+(1-2 \zeta) \sinh \alpha]}\right) .
\end{aligned}
$$

In the MLLA approximation, the spectrum for massless quark jets differs by an overall prefactor $C_{F} / N_{c}=4 / 9$ only. Here, $I_{B}$ denotes the modified Bessel function of order $B$ and the factors $A, B$ are determined by the prefactors of the LO parton splitting functions $P_{q \rightarrow q g}, P_{g \rightarrow g g}$ and $P_{g \rightarrow q q}$,

$$
A=\frac{4 N_{c}}{b}, \quad B=\left(\frac{11}{3} N_{c}+\frac{2}{3} \frac{n_{f}}{N_{c}^{2}}\right) / b, \quad b=\frac{11}{3} N_{c}-\frac{2}{3} n_{f},
$$

where $N_{c}$ is the number of colors and $n_{f}$ the number of flavors. In what follows we use $N_{c}=n_{f}=3$. We have introduced also the following notational shorthands

$$
Y=\ln \frac{E_{\text {jet }}}{\Lambda}, \quad \alpha=\alpha_{0}+i \tau, \quad \tanh \alpha_{0}=2 \zeta-1, \quad \zeta=1-\frac{\xi}{Y} .
$$

Local parton hadron duality (LPHD) [51, 53, 54] is a model which translates the partonic yield (2.1) into a hadronic one. For unidentified hadrons, LPHD postulates a one-to-one correspondence between partons and hadrons, introducing a proportionality factor of order $O(1)$

$$
\frac{d N^{\text {hadrons }}}{d \xi}=K_{\mathrm{LPHD}} D_{q, g}(\xi, Y, \lambda) \text {. }
$$

To describe the spectra of identified hadrons inside jets, it has been suggested to go beyond the limiting spectrum (2.1) by stopping the evolution of the parton shower at a finite scale $\lambda$, related 
(a)

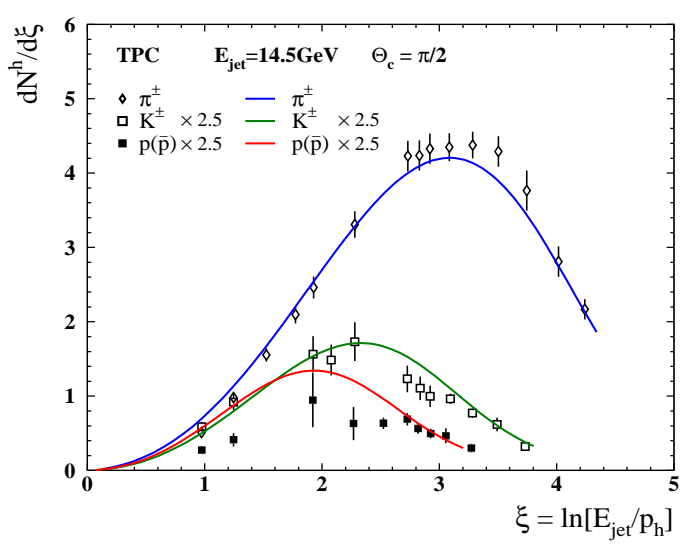

(b)

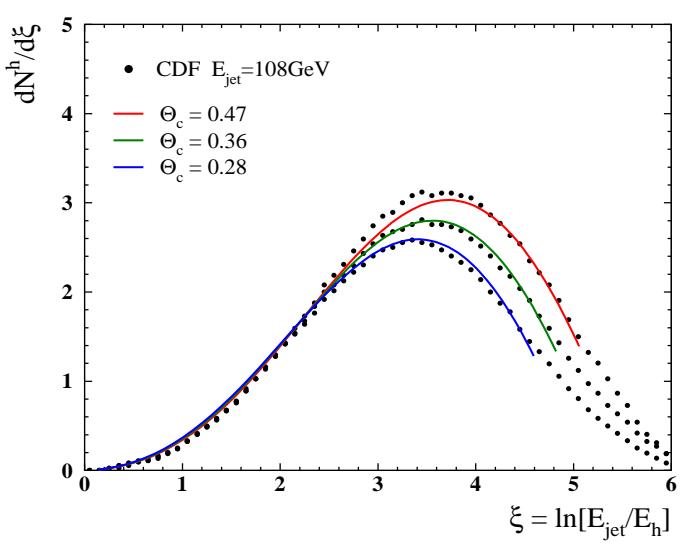

FIG. 2: Two examples for the comparison of the MLLA+LPHD formalism with data on single inclusive spectra inside a jet as a function of the logarithm of the hadron momentum fraction $\xi$. a) The distribution (2.4) of charged pions $\left(\pi^{+}+\pi^{-}\right)$, kaons $\left(K^{+}+K^{-}\right)$and (anti)-protons $(p+\bar{p})$ in a jet of energy $E_{\text {jet }}=14.5 \mathrm{GeV}$, compared to TPC data on $e^{+} e^{-}$collisions [55]. The MLLA parameters are $\Lambda=155 \mathrm{MeV}$ and $K_{\mathrm{LPHD}}=1.22$. b) The MLLA+LPHD distribution of all charged hadrons in a jet of energy $E_{\text {jet }}=108 \mathrm{GeV}$ for various opening angles $\Theta_{c}$, compared to CDF data from $p \bar{p}$ collisions [56]. The MLLA parameters are $\Lambda=Q_{0}=235 \mathrm{MeV}, K_{\mathrm{LPHD}}=0.555$.

to the mass of the hadron $Q_{0} \approx M_{h}[53,54]$. This leads to

$$
D^{h}\left(\bar{\zeta}, E_{\text {jet }}, M_{h}\right)=K_{0}\left(M_{h}\right) D^{\lim }\left(\bar{\zeta}, E_{\text {jet }}\right),
$$

where

$$
K_{0}\left(M_{h}\right)=\frac{2}{\Gamma(B)}(A \lambda)^{B / 2} K_{B}(\sqrt{4 A \lambda}) .
$$

Here, $K_{B}$ is the modified Bessel function of order $B$ and

$$
\bar{\zeta}=\frac{y}{y_{\max }}, \quad y=\ln \frac{E_{h}+p_{h}}{M_{h}} .
$$

In Fig. 2a a, we compare the distribution (2.5) with single inclusive spectra of identified hadrons, measured in jets in $e^{+} e^{-}$collisions. We use the mass $M_{h}$ of the identified hadron species in the definition of the rapidity (2.7) and in the argument of the prefactor $K_{0}$ in (2.6) , Here, the limiting spectrum was calculated taking $Q_{0}=\Lambda \approx M_{\pi}$. In accordance with data, one observes that the spectrum gets harder for more massive hadrons. Also, the mass-dependent hierarchy of hadron multiplicity is reproduced. For an improved agreement, we followed [54] and multiplied the conversion factor $K_{\mathrm{LPHD}}$ by an additional suppression factor $\gamma_{s}=0.73$ for kaons. This heuristic factor may account for the fact that the probability of hadronizing into strange hadrons is reduced due to the larger mass of the strange quark. In contrast to the original analysis [54], we calculate $K_{0}\left(M_{h}\right)$ from (2.6) rather than extracting it from a fit to TPC data.

In Fig. 2b, we compare the MLLA formalism to the single inclusive spectra of all charged hadrons, contained inside smaller jet subcones of opening angles $\Theta_{c}$. The MLLA formalism is the result of an evolution equation which implements a parton shower with angular ordering, and the $\Theta_{c^{-}}$-dependence of $D^{h}$ is given by replacing in (2.3)

$$
E_{\text {jet }} \longrightarrow E_{\text {jet }} \sin \Theta_{c} \text {. }
$$


(a)

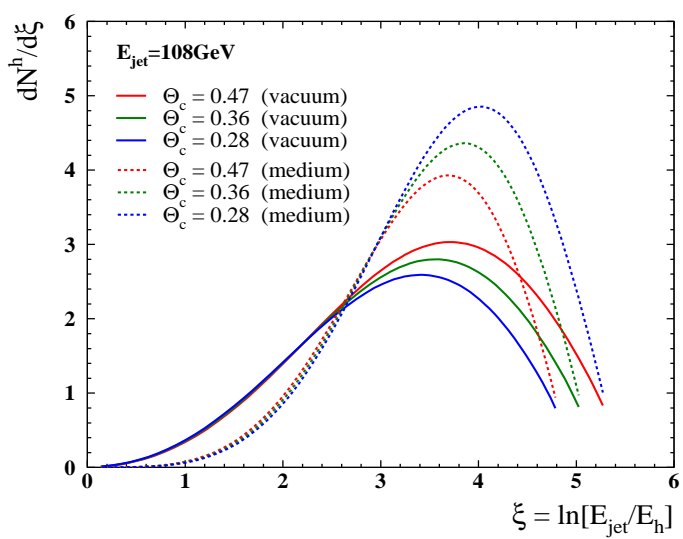

(b)

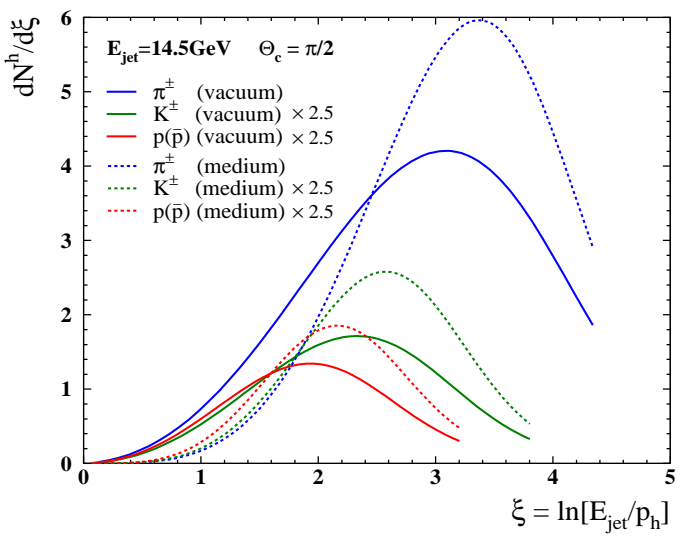

FIG. 3: Medium modification of the single inclusive spectra inside a jet as a function of the logarithm of the hadron momentum fraction $\xi$ for $f_{\text {med }}=1$. a) Distributions of all charged particles in the jet of energy $E_{\text {jet }}=108 \mathrm{GeV}$ for various opening angles $\Theta_{c}$. b) Expected modification of the pion, kaon and proton spectra in the jet of energy $E_{\text {jet }}=14.5 \mathrm{GeV}$ and opening angle $\Theta_{c}=\pi / 2$.

As seen in Fig. 2b, and in accordance with [56], the MLLA formalism leads to a fair description of the $\Theta_{c}$-dependence of the unidentified charged hadron spectrum $d N^{h} / d \xi$ inside a jet. (We note as an aside that the $\mathrm{CDF}$ data in Fig. $2 \mathrm{~b}$ are plotted versus the variable $\ln \left[E_{\text {jet }} / E_{h}\right]$. Hence, we have calculated the distributions in Fig. $2 \mathrm{~b}$ and Fig. [3a as a function of $\ln \left[E_{\text {jet }} / E_{h}\right]$, which we also denote by a slight abuse of notation with the variable $\xi$. Everywhere else in this paper, we use $\xi=\ln \left[E_{\mathrm{jet}} / p_{h}\right]$.) We are not aware of an experimental study of the jet hadrochemical composition as a function of the jet opening angle. In what follows, we assume that the relative distributions of identified hadron species inside a jet do not change significantly as a function of $\Theta_{c}$, so that the replacements (2.8) applies to identified yields, too.

In summary, MLLA+LPHD are known to provide a fair description of the charged and identified single inclusive hadron spectra inside a jet, and of their dependence on jet opening angle. This will serve as the baseline for the following study of jet medium modifications.

\section{B. Modeling medium modifications of single inclusive intrajet distributions}

A unique prescription of how to model the medium modification of jet fragmentation does not exist. RHIC data indicate that a realistic prescription should lead to a softening of the jet distribution, consistent with a suppression of the leading hadron spectra by a factor $\sim 5$ in central $\mathrm{Au}+\mathrm{Au}$ collisions. One possibility to achieve this is to enhance the probability of parton branching in the jet fragmentation. This is also motivated by calculations of medium-induced gluon radiation of hard partons, which imply enhanced parton splitting. In what follows, we consider a model of this type [27], in which the singular parts of all parton splitting functions $P_{q q}, P_{g g}$ and $P_{g q}$ are enhanced by one common model-dependent factor $\left(1+f_{\text {med }}\right)$, such that for instance

$$
P_{q q}=C_{F}\left\{\frac{2\left(1+f_{\text {med }}\right)}{(1-z)_{+}}-(1+z)\right\} .
$$

The factor $\left(1+f_{\text {med }}\right)$ is the only medium modification in our model. The LPHD-prescription will be adopted unchanged. For the present exploratory study, this model has several wanted features: The model is easy to implement. Within the MLLA formalism, the medium-modification (2.9) 
amounts solely to a redefinition of the parameters $A$ and $B$,

$$
A=\frac{4 N_{c}\left(1+f_{\mathrm{med}}\right)}{b}, \quad B=\left(\frac{11+12 f_{\mathrm{med}}}{3} N_{c}+\frac{2}{3} \frac{n_{f}}{N_{c}^{2}}\right) / b .
$$

The model describes not only leading fragments, but the entire jet distribution. Also, the value of the only model parameter $f_{\text {med }}$ can be constrained since it determines the degree to which single inclusive hadron spectra are suppressed. Here, we add the caveat that the MLLA spectrum becomes unreliable in the region of large momentum fraction, $\xi<1$ say, mainly since MLLA resums only logarithms in $1 / x$ and not in $1 /(1-x)$. The use of $D^{h}\left(\xi, Q=E_{\text {jet }}\right)$ as a fragmentation function for leading hadron production is thus unreliable. But the finding that in this way a value $f_{\text {med }}=0.6-0.8$ can account for a suppression $\sim 5$ of leading hadron spectra, may still provide an indication of the parameter range of $f_{\text {med }}$ supported by data [27]. Our only reason for mentioning this argument is to motivate a choice of $f_{\text {med }}$. It is clear that at the LHC, experimental constraints on $f_{\text {med }}$ will come mainly from measuring the distributions shown in Fig. 3, rather than from single inclusive hadron spectra. In the absence of such constraints, we use for the following numerical studies $f_{\text {med }}=1$, which lies certainly in the right order of magnitude, and allows us to illustrate the features of this model.

We now discuss the modifications of jet observables introduced by this model. As seen in Fig. [3a, enhancing the parton splitting by a factor $\left(1+f_{\text {med }}\right)$ softens the jet multiplicity distributions irrespective of the jet opening angle. Also, this softening is reflected in all identified hadron spectra, see Fig. 3 b. The mass hierarchy of the intrajet distributions is preserved in this model: the yields of heavier hadrons peak at larger momentum fractions and thus at smaller $\xi$.

To better characterize the medium modification of the jet hadrochemical composition, implemented in this model, we focus in the following not on the absolute yields as in Fig. 3, but on the ratios of identified hadron yields. Also, we translate the $\xi$-dependence at fixed $E_{\text {jet }}$ into a transverse momentum dependence. For a single jet of energy $E_{\text {jet }}$, the $p_{T}$-spectrum of identified hadronic fragments of type $h$, collected within the opening angle $\Theta_{c}$, takes the form

$$
\left[\frac{d N^{h}\left(\Theta_{c}\right)}{d p_{T}}\right]_{\text {jet }}=K_{\mathrm{LPHD}} \gamma_{h} K_{0}\left(M_{h}\right) \frac{1}{p_{T}} D^{\lim }\left(\bar{\zeta}\left(p_{T}, M_{h}, E_{\text {jet }}\right), E_{\text {jet }}, \Theta_{c}, \Lambda\right) .
$$

Here, $\gamma_{h}$ is an additional particle species dependent suppression factor. We choose $\gamma_{h}=1$ for pions and protons, and $\gamma_{K}=\gamma_{s}=0.73$ for kaons [54], which are the same choices as made in section IIA] For the local parton-hadron duality parameter, we take $K_{\mathrm{LPHD}}=0.5$. This factor must be slightly lower than the one used in Fig. 2 $\mathrm{b}$, since it determines the normalization of the identified hadron spectra, while Fig. 2 $\mathrm{b}$ shows the spectrum of all charged particles. We note that for $f_{\text {med }}=1$, $K_{0}\left(M_{h}\right)$ changes only by up to $\sim 12 \%$ from its value in the vacuum. In the following, we shall focus on results for a relatively small opening angle $\Theta_{c}=0.28 \mathrm{rad}$. We have tested that the dependence of identified particle ratios within the jet on $\Theta_{c}$ is very weak .

In our model, the hadrochemical composition of jet fragments changes significantly in the presence of parton energy loss (i.e. for finite $f_{\text {med }}$ ). Heavier hadrons become more abundant. As seen

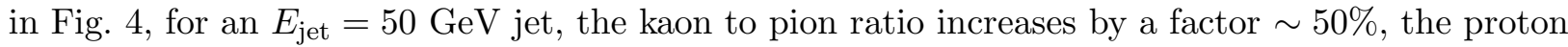
to pion ratio by a factor $\sim 100 \%$. These medium-induced changes persist over the entire transverse momentum range. They decrease slightly with increasing jet energy, but remain clearly visible even for $E_{\text {jet }}=200 \mathrm{GeV}$ jets.

The significant medium modification of jet hadrochemistry is remarkable, since the present model does not encode for medium effects at or after hadronization. Also, in contrast to the sketch in Fig. 1, the model does not involve color transfer between projectile and target, nor does it involve the transfer of other quantum numbers. It only encodes an enhancement of the probability 


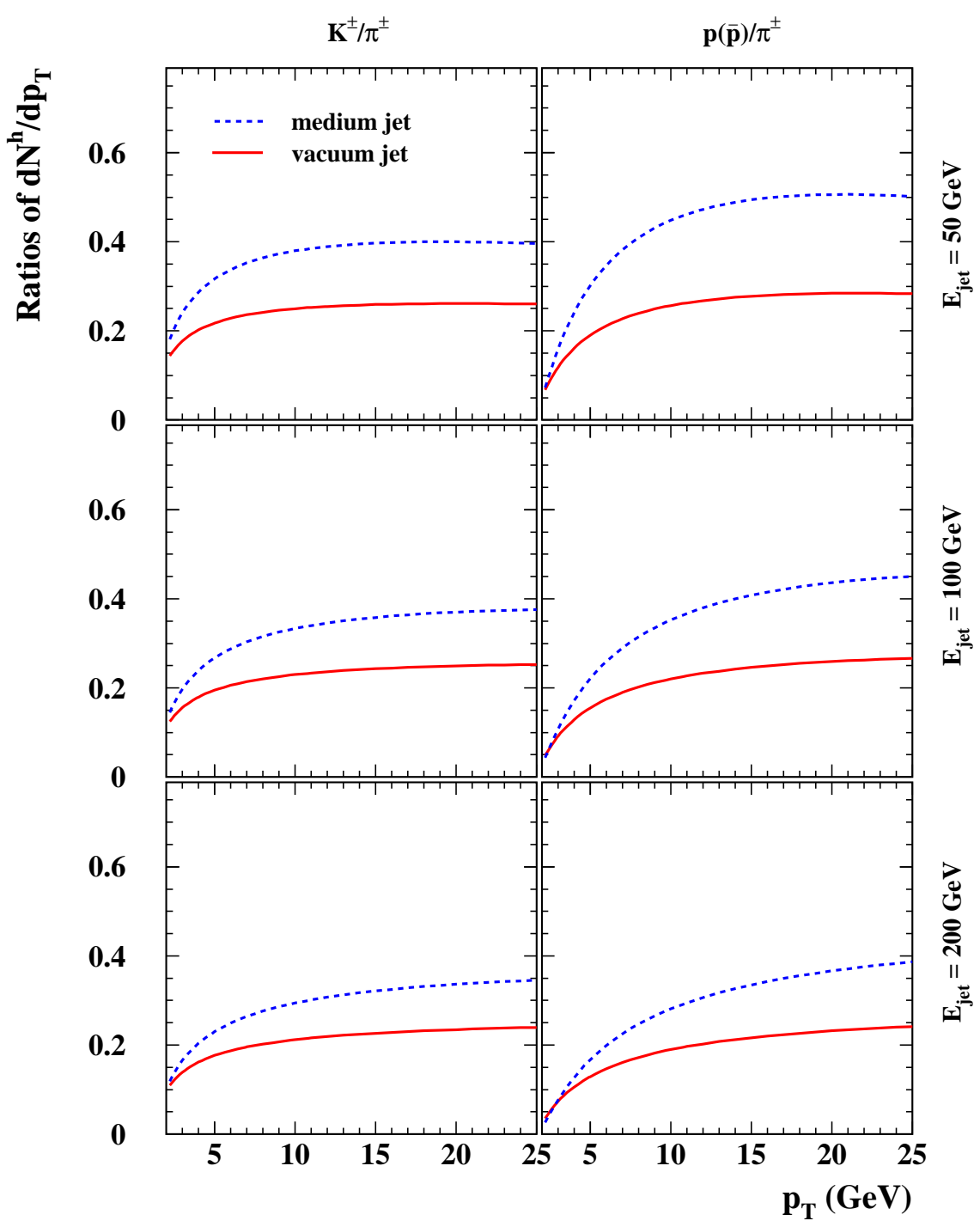

FIG. 4: Results of the MLLA+LPHD formalism for $K^{ \pm} / \pi^{ \pm}$and $p(\bar{p}) / \pi^{ \pm}$ratios in jets with energies $E_{\text {jet }}=$ 50, 100 and $200 \mathrm{GeV}$. The jet opening angle is $\Theta_{c}=0.28$ and medium-induced changes are calculated for $f_{\text {med }}=1$.

of parton splitting, which affects the distribution of the invariant mass of partons at the end of the parton shower. It is one of the main results of this paper, that enhanced parton splitting alone without explicit medium-induced modification of the hadronization mechanism can lead to significant changes in the jet hadrochemical composition. In this sense, characteristic deviations in jet hadrochemistry are a generic characteristics of any parton energy loss mechanism, as long as medium-modified splitting is indispensable for parton energy loss.

We have studied whether medium effects generally enhance the yield of heavier hadrons, as seen in Fig. 4, or whether the opposite may be possible. In the context of MLLA+LPHD, we observe that the yield of heavier hadrons peaks at smaller values of $\xi$ and that increasing the splitting parameter $f_{\text {med }}$ enhances the soft part of this yield. However, the $\xi$-region which is soft for a heavy hadron is still hard for a light hadron such as a pion. For instance, in Fig. $3 \mathrm{~b}$, this is the case for $1.5<\xi<2.5$, since the pion yield decreases with increasing $f_{\text {med }}$ for $\xi<2.5$, while 
the proton yield increases for $\xi>1.5$. This opposite $f_{\text {med }}$-dependence at intermediate $\xi$ excludes the possibility that the ratios $K^{ \pm} / \pi^{ \pm}$and $p(\bar{p}) / \pi^{ \pm}$decrease over the entire transverse momentum range with increasing medium-effects. This gives support to the idea that the enhancement of heavier hadrons, observed in Fig. 4, is rather generic for jet quenching models.

\section{MEDIUM-MODIFIED JETS IN A HIGH-MULTIPLICITY ENVIRONMENT}

The success of MLLA+LPHD in reproducing hadrochemical distributions (see e.g. Fig. 22a), as well as the model of medium modifications described in section IIB, are based on a mass effect: Identified hadronic yields are determined by relating the QCD evolution scale to the mass of the hadron species. In contrast, RHIC data indicate that the hadrochemical abundance of the underlying event in heavy ion collisions follows a valence quark counting rule in a wide intermediate transverse momentum range: The identified hadronic yields are characterized by a baryon-tomeson enhancement, irrespective of the hadron mass [1, 4]. So, the hadrochemical composition of unmodified jets, and of the underlying event in heavy ion collisions appear to differ qualitatively.

If parton energy loss constitutes the onset of a partonic equilibration mechanism, does this imply that the hadrochemical distribution of medium-modified jets approaches that of the medium, rather than remaining qualitatively different? Access to these and other far reaching questions may be gained by establishing whether the qualitative difference between jet hadrochemistry and bulk hadrochemistry persists at the LHC. Here, we shall not attempt to address these questions by building jet quenching models which implement specific features of hadrochemical equilibration. Rather, we explore in a jet quenching model without hadrochemical equilibration (namely that of section (IB), what happens if the particle identified yield of a jet is superimposed to a realistic heavy ion background. This may serve as a baseline on top of which signals of hadrochemical equilibration can be established. We first introduce in section III A a recombination model for the underlying event in heavy ion collisions. Then we superimpose in section IIIB jet multiplicity distributions to this underlying event.

\section{A. Two component model of underlying event}

To model the underlying event we use the framework proposed in [39, 43] and further explored in [4]], in which hadrons are produced via two competing mechanisms, recombination and fragmentation. Recombination models assume that hadrons can form by coalescence of constituents quarks, which are taken as effective degrees of freedom during hadronization. The recombination mechanism competes with the standard fragmentation of quarks into hadrons. Recombination models typically discard gluonic degrees of freedom and use the same quark spectrum for the calculation of recombination and fragmentation contributions to single inclusive spectra. Despite these simplifications, a fair description of relative hadrochemical yields is obtained [43, 44]. Here, we limit ourselves to central collisions and we specify the model input following Ref. [44]. The main input for recombination models is knowledge about the partonic transverse momentum spectrum. This is typically modeled by a two-component distribution, showing an exponential "thermal" slope at low transverse momentum and displaying a characteristic power-law at high transverse momentum.

We characterize the exponential component of the quark and anti-quark spectrum by the distribution

$$
w_{i}(R, p) \sim e^{-p^{\mu} v_{\mu}(R) / T} .
$$

This distribution is assumed to be emitted from spatio-temporal positions $R^{\mu}=$ $(\tau \cosh \eta, \rho \cos \phi, \rho \sin \phi, \tau \sinh \eta)$, which lie in a thermally equilibrated system at temperature $T$ 
along a space-like freeze-out hypersurface $\Sigma$. Here, $\eta$ is the space-time rapidity, $\rho$ the radial coordinate, and a suitable hypersurface can be specified by fixing $\tau=\sqrt{t^{2}-z^{2}}=$ const. The system is expanding at position $R^{\mu}$ with a longitudinally boost-invariant flow profile $v_{\mu}(R)$, which displays a velocity $v_{T}=\tanh \eta_{T}$ in the transverse direction. Integrating $w_{i}$ along the freeze-out hypersurface, it is a standard procedure to find the soft contribution to the quark spectrum, $d N_{a}^{\text {soft }} / d^{2} p_{a, T} d y$. In the following, we denote by $p_{a, T}$ the momentum of partons, and by $p_{T}$ the momentum of hadrons.

The hard, power-law contribution to the quark spectrum is determined by

$$
\left.\frac{d N_{a}^{\text {hard }}}{d^{2} p_{a, T} d y}\right|_{y=0}=K \frac{C}{\left(1+p_{a, T} / B\right)^{\beta}} .
$$

Here, the parameters $C, B$ and $\beta$ are taken from leading order perturbative QCD calculations [57] and the constant $K \simeq 1.5$ accounts for higher order corrections [39, 43]. Parton energy loss is modeled by quenching this partonic spectrum via shifting its momentum distribution by $\Delta p_{a, T}\left(p_{a, T}\right)=\epsilon_{0} \sqrt{p_{a, T}}$, as suggested in [58].

We now explain how these partonic spectra are turned into hadronic yields. For an exponential spectrum based on (3.1), recombination always wins over fragmentation, since there are exponentially many recombination partners at soft $p_{T}$. For a power-law tail (3.2), however, fragmentation wins over recombination, since there are sufficiently many high- $p_{T}$ components which can fragment into softer ones. Thus, the partonic $p_{a, T}$-scale at which the power-law contribution (3.2) overcomes the exponential one sets the hadronic $p_{T}$-scale at which fragmentation starts to dominate over recombination [43].

The momentum spectrum for mesons and baryons from recombination can be written as [43, 44]

$$
\left.\frac{d N_{M, B}}{d^{2} p_{T} d y}\right|_{y=0}=C_{M, B} M_{T} \frac{\tau A_{T}}{(2 \pi)^{3}} 2 \Pi_{a} \gamma_{a} I_{0}\left[\frac{p_{T} \sinh \eta_{T}}{T}\right] k_{2,3}\left(p_{T}\right),
$$

where $\gamma_{a}$ are quark fugacities, $C_{M, B}$ the degeneracy factors for mesons and baryons respectively, and $M_{T}$ their transverse masses. $A_{T}=\pi \rho_{0}^{2}$ is the transverse area of the parton system at freeze-out and $\tau$ the hadronization time. Here, we introduced the shorthand $k_{N}\left(p_{T}\right)=$ $K_{1}\left[\frac{\cosh \eta_{T}}{T} \sum_{a=1}^{N} \sqrt{m_{a}^{2}+\frac{p_{T}^{2}}{N^{2}}}\right]$.

The spectrum for hadrons from fragmentation is given by

$$
E \frac{d N_{h}}{d^{3} p_{T}}=\sum_{a} \int_{0}^{1} \frac{d z}{z^{2}} D_{a \rightarrow h}\left(z, Q^{2}\right) E_{a} \frac{d N_{a}^{\text {hard }}}{d^{3} p_{a, T}}
$$

with $D_{a \rightarrow h}\left(z, Q^{2}\right)$ denoting the fragmentation function of a parton $a$ into a hadron $h$. We use KKP fragmentation functions $[59]$.

It has been shown [39, 43, 44] that with appropriately chosen parameters, this two component model accounts successfully for the baryon-to-meson enhancement observed in a large class of RHIC data on $\mathrm{Au}+\mathrm{Au}$ collisions at intermediate $p_{T}$. In particular, recombination models can reproduce the proton to pion and kaon to pion ratio at intermediate transverse momentum [39, 43, 44]. Recombination dominates at RHIC up to $p_{T}^{\text {hadron }} \simeq 4-6 \mathrm{GeV}$, and fragmentation takes over for higher transverse momentum.

This model has been extrapolated to $\mathrm{Pb}+\mathrm{Pb}$ collisions at $\sqrt{s}=5.5 \mathrm{TeV}$ [44, 45] by fixing the temperature of the quark phase at hadronization at $175 \mathrm{MeV}$, similar to the RHIC case, and rescaling the parameters $v_{T}$ and $\tau A_{T}$ such that the results of fluid simulations [60] are reproduced: $v_{T}=0.68$ and $\tau A_{T}=11.5 \times 10^{3} \mathrm{fm}^{3}$ [44]. The quenching of high- $p_{a, T}$ partons is fixed by the choice $\epsilon_{0}=2.5$, which amounts to a factor $\simeq 10$ suppression of the single inclusive hadron spectra at 


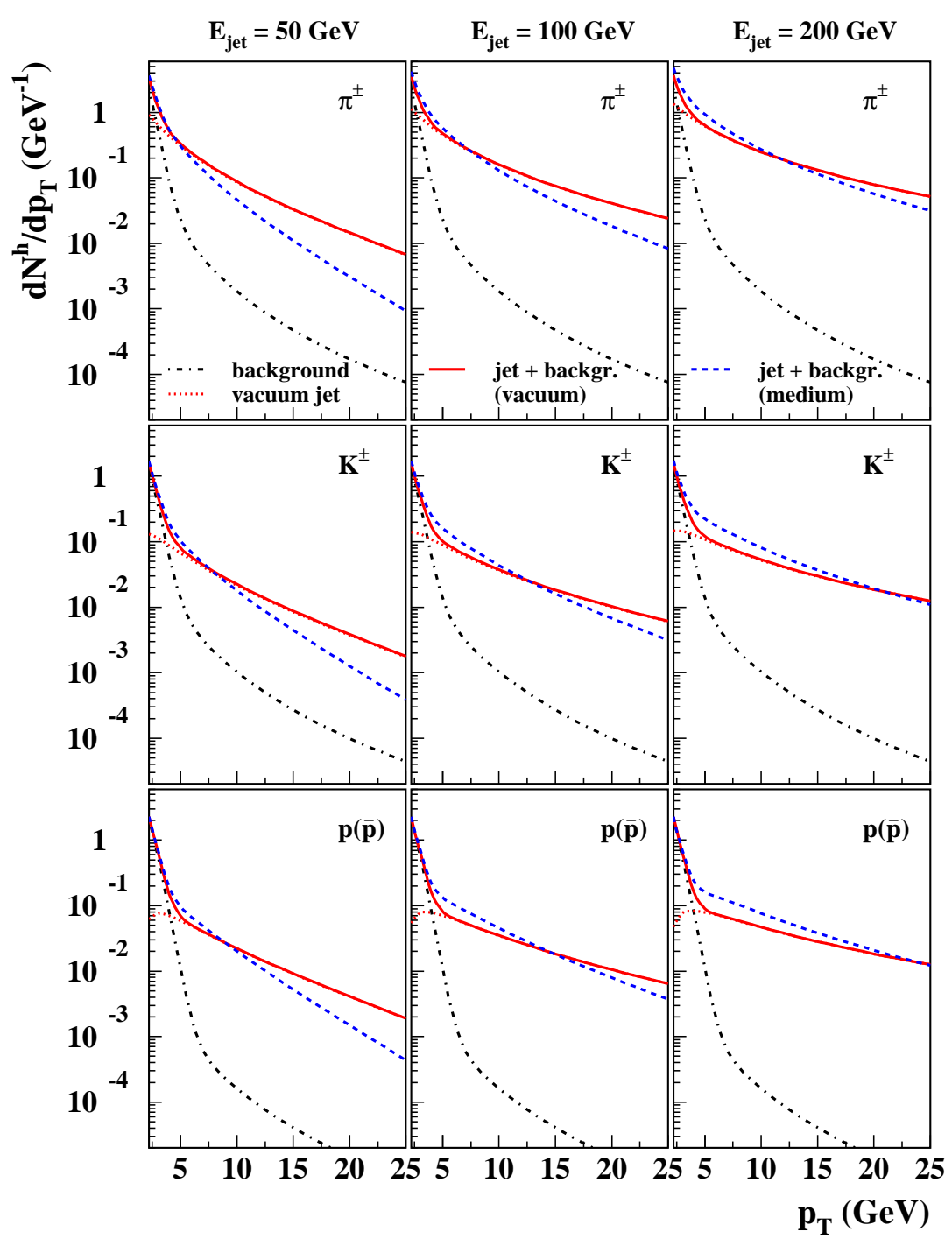

FIG. 5: Identified transverse momentum spectra within a cone of opening angle $\Theta_{c}=0.28$ for pions, kaons and protons.

$p_{T}=10 \mathrm{GeV}$. Our description of the hadrochemical composition of the underlying event in heavy ion collisions is based on this model. The single inclusive hadron spectra calculated such for LHC are dominated by recombination up to a scale which lies $\simeq 2 \mathrm{GeV}$ higher than the corresponding scale at RHIC [44, 45].

\section{B. The hadrochemical composition of jets within high-multiplicity nucleus-nucleus collisions}

In this section, we superimpose the jet spectrum calculated in section II onto the background spectrum introduced in section IIIA. The background yield in a cone of opening angle $\Theta_{c}$ is given by

$$
\left.\left[\frac{d N^{h}\left(\Theta_{c}\right)}{d p_{T}}\right]_{\text {background }} \simeq \Theta_{c}^{2} \pi p_{T} \frac{d N^{h}}{d^{2} p_{T} d y}\right|_{y=0}
$$


where the spectrum on the right hand side is a sum of contributions from recombination (3.3) and fragmentation (3.4). To arrive at this expression, we have integrated the full double-differential spectrum $d N^{h} /\left.d^{2} p_{T} d y\right|_{y=0}$ over one unit in rapidity and the full azimuthal phase space. Since the spectrum is flat around mid-rapidity, the integral is trivial. In the $\Delta \eta \times \Delta \phi$-space, this is an area of $2 \pi$. We have then multiplied our result with the fraction $\pi \Theta_{c}^{2} / 2 \pi$ which a cone of opening angle $\Theta_{c}$ occupies in this plane.

A jet of energy $E_{\text {jet }}$ will deposit within the opening angle $\Theta_{c}$ the particle yield $\frac{d N^{h}\left(\Theta_{c}\right)}{d p_{T}}$ given in Eq. (2.11). On average, this jet will sit on top of the background (3.5). In Fig. 5, we compare these two contributions. One sees that despite the high multiplicity environment of a heavy ion collision, the harder distribution of jet fragments dominates rapidly over the abundant distribution of soft particles at transverse momenta larger than $5-7 \mathrm{GeV}$. In the high- $p_{T}$ region, the slope of the combined transverse momentum spectrum is dominated by jet fragments. This slope steepens characteristically in the presence of medium-induced parton energy loss. Hence, if the energy of the jet can be measured reliably in heavy ion collisions, then such transverse momentum spectra provide direct experimental access to the longitudinal jet fragmentation function.

In Fig. 6, we have plotted the identified particle ratios $K^{ \pm} / \pi^{ \pm}$and $p(\bar{p}) / \pi^{ \pm}$, measured in a cone of opening angle $\Theta_{c}$. From an experimental point of view, this may be a relatively straightforward hadrochemical measurement: It is performed by counting all particles within an opening angle $\Theta_{c}$ as a function of $p_{T}$. Particle identification is statistical. An experimental separation of the jet from the underlying event is not needed. In principle, the measurement requires knowledge of the energy of the jet contained in the opening angle. However, this determination of $E_{\text {jet }}$ does not need to be particularly good, since the hadronic ratios shown in Fig. 6 depend only weakly on $E_{\text {jet }}$.

We remarked in our discussion of Fig. 5 that in the presence of a jet, the spectrum within an opening cone is essentially background free above $p_{T}>5-7 \mathrm{GeV}$. In Fig. 6, this is reflected in the fact that above this transverse momentum scale, the particle ratios match those shown in Fig. 4 and calculated without including the background. It is remarkable that in this high- $p_{T}$ range, medium-effects enhance these ratios, thus widening rather than narrowing the difference with the background. At lower transverse momentum, the background yield dominates the hadronic abundances and particle ratios.

We finally explore yet another possibility of presenting information about the hadrochemical composition in jets and their change in a medium. To this end, we introduce, in close analogy with the nuclear modification factor, the jet modification factor

$$
J_{\mathrm{AA}} \equiv \frac{\left.\frac{d N^{h}}{d p_{T}}\right|_{\mathrm{med}}}{\left.\frac{d N^{h}}{d p_{T}}\right|_{\mathrm{vac}}} .
$$

Here, the numerator denotes the particle spectrum within a cone of opening angle $\Theta_{c}$ in the presence of a jet. We determine it as the sum of the background (3.5) and the spectrum (2.11) for a quenched jet $\left(f_{\text {med }}=1\right)$. The denominator is constructed experimentally by measuring the minimum bias spectrum in a cone of angle $\Theta_{c}$ and adding the spectrum of an unquenched jet. In our calculation, we add on top of the background (3.5) the spectrum (2.11) for a vacuum jet $\left(f_{\text {med }}=0\right)$.

Jet quenching amounts to a reshuffling of hadronic yield from high to low transverse momentum. The transverse momentum scale $p_{T}^{\text {crit }}$ above which the yield in a jet spectrum is depleted is characterized in the measurement of $J_{\mathrm{AA}}$ by $J_{\mathrm{AA}}\left(p_{T}^{\text {crit }}\right)=1$. From the right column of Fig. 7 , one sees that $p_{T}^{\text {crit }}$ grows significantly with $E_{\text {jet }}$. This indicates that the subleading jet fragments, additionally produced due to medium effects, are distributed in an increasingly wide transverse momentum regime which extends significantly beyond the low- $p_{T}$ region dominated by background yield, and up to $p_{T}^{\text {crit }}$. Also, the total amount of additional multiplicity, produced due to parton energy loss, increases with $E_{\text {jet }}$, and so $J_{\mathrm{AA}}$ increases with $E_{\text {jet }}$ in a wide intermediate transverse momentum regime in 


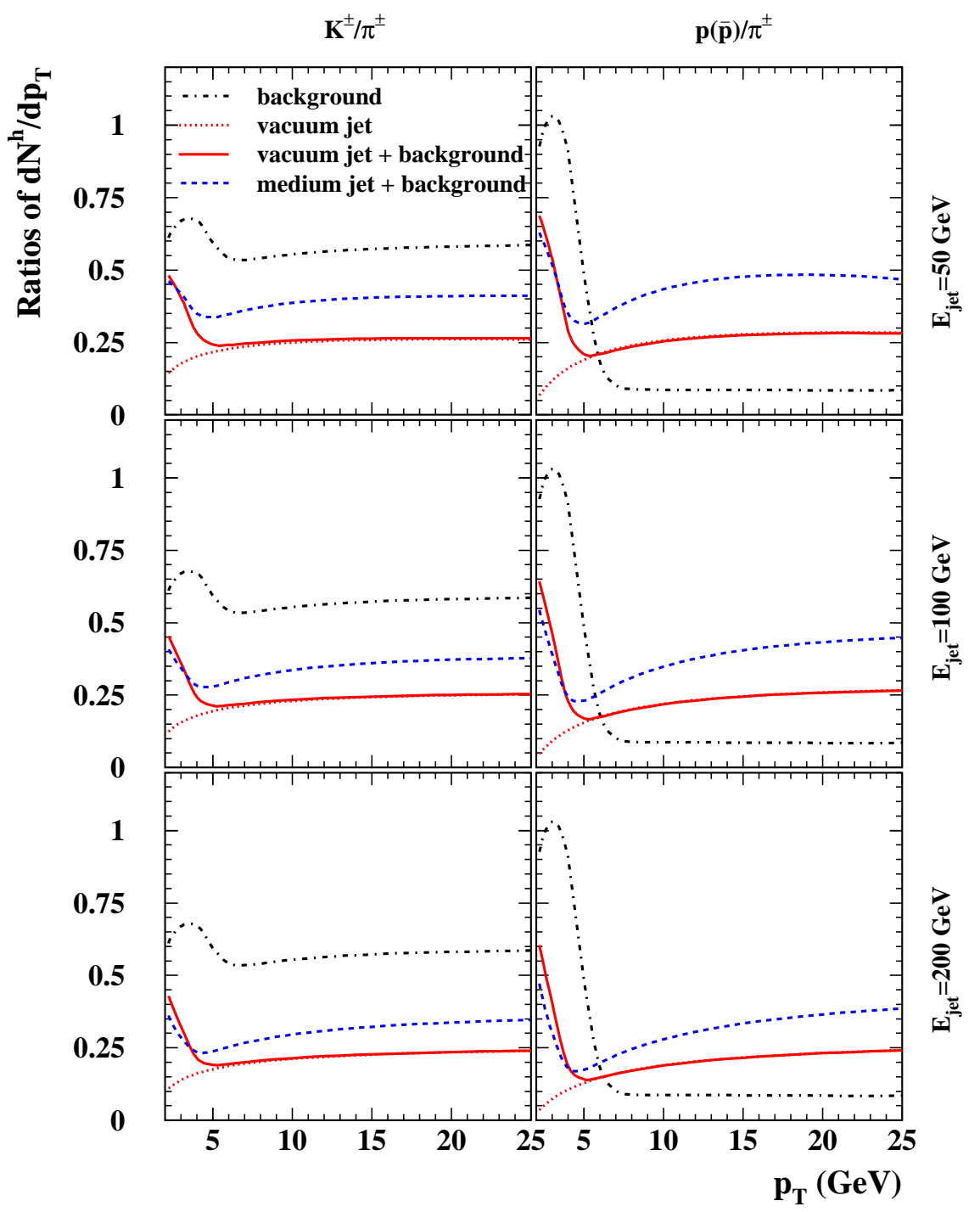

FIG. 6: The particle ratios $K^{ \pm} / \pi^{ \pm}$and $p(\bar{p}) / \pi^{ \pm}$obtained from the spectra shown in Fig. 5 . These ratios are measured in a cone of opening angle $\Theta_{c}=0.28$ in the $\Delta \eta \times \Delta \phi$-plane, which contains both soft background and a jet of energy $E_{\text {jet }}$.

which hadrochemical identification is possible. The order of the particle species dependence of $J_{\mathrm{AA}}$, seen in the left column of Fig. 7, is a direct consequence of the medium-induced enhancement of the ratios $K^{ \pm} / \pi^{ \pm}$and $p(\bar{p}) / \pi^{ \pm}$, seen in Figs. 5 and 6 . Namely, if for instance at fixed transverse momentum, $\left.\left\langle K^{ \pm}\right\rangle_{\text {med }} /\left\langle\pi^{ \pm}\right\rangle_{\text {med }}\right\rangle\left\langle K^{ \pm}\right\rangle_{\text {vac }} /\left\langle\pi^{ \pm}\right\rangle_{\text {vac }}$, then $\left.\left\langle K^{ \pm}\right\rangle_{\text {med }} /\left\langle K^{ \pm}\right\rangle_{\text {vac }}\right\rangle\left\langle\pi^{ \pm}\right\rangle_{\text {med }} /\left\langle\pi^{ \pm}\right\rangle_{\text {vac }}$, and this order is reflected in Fig. 7 .

\section{SUMMARY}

The analysis of the hadrochemical composition of jets in heavy ion collisions is a terra incognita, for which data are not available yet. It is in experimental reach of the LHC. In this paper, we have argued that jet quenching generically implies modifications of the jet hadrochemical composition (see discussion in sections 1 and IB ). A simple model of a parton shower with enhanced medium- 


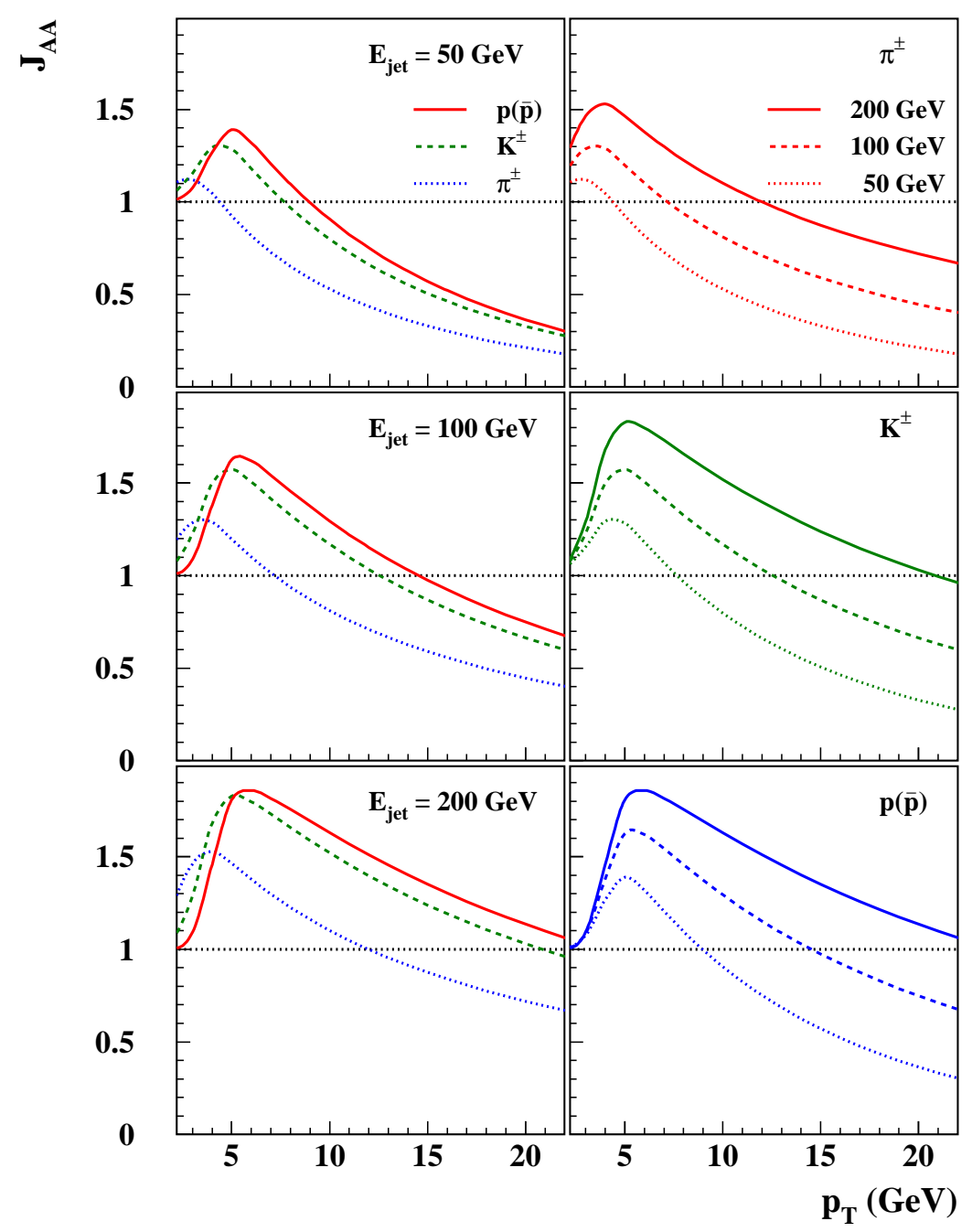

FIG. 7: The jet modification factor $J_{A A}$, defined in (3.6) within a cone of opening angle $\Theta_{c}=0.28$ as a function of transverse momentum for different jet energies and different hadron species.

induced branching but unmodified hadronization (see section IIB) then allowed us to illustrate that a significantly modified jet hadrochemistry can be expected even if at the time of hadronization, medium effects are not present any more. This is important, since formation time arguments indicate that for sufficiently large $E_{\text {jet }}$, hadronization occurs outside the medium produced in heavy ion collisions.

Remarkably, at sufficiently high $p_{T}$, we observed that medium modifications increase the hadrochemical ratios $K^{ \pm} / \pi^{ \pm}$and $p(\bar{p}) / \pi^{ \pm}$, thus further increasing the difference between the distributions inside the jet and those of the underlying event. We gave arguments of why this is likely to be a generic feature, which should persist for a wide class of jet quenching models. On the other hand, any jet quenching mechanism, which for instance kicks components of the background into the jet cone, may be expected to have the opposite effect, namely to narrow the difference between the hadrochemical composition of the jet and the background. It is in this sense, that we view the model study presented in sections $\amalg \mathrm{I}$ and $\amalg$ as a baseline, on top of which effects indicative of specific microscopic mechanisms of parton energy loss may be established. 


\section{Acknowledgments}

We have profited from discussions with Nicolas Borghini, Yuri Dokshitzer, Krzysztof GolecBiernat, Andreas Morsch, Karel Safarik, Jürgen Schukraft, and Yves Schutz. We thank Steffen Bass, Rainer Fries and Carlos Salgado for sharing with us the results of simulations, documented in Refs. [43, 44]. We acknowledge support from the Marie Curie ESRT Fellowship of the European Community's Sixth Framework Programme under contract number (MEST-CT-2005-020238), which made this project at CERN possible. One of us (S.S.) also acknowledges a grant of the Polish Ministry of Science, N202 048 31/2647 (2006-08).

[1] K. Adcox et al. [PHENIX Collaboration], Nucl. Phys. A 757 (2005) 184.

[2] B. B. Back et al. [PHOBOS Collaboration], Nucl. Phys. A 757 (2005) 28.

[3] I. Arsene et al. [BRAHMS Collaboration], Nucl. Phys. A 757 (2005) 1.

[4] J. Adams et al. [STAR Collaboration], Nucl. Phys. A 757 (2005) 102.

[5] F. Carminati et al. [ALICE Collaboration], J. Phys. G 30 (2004) 1517.

[6] B. Alessandro et al. [ALICE Collaboration], J. Phys. G 32 (2006) 1295.

[7] D. D'Enterria et al., CERN-LHCC-2007-009, J. Phys. G in press.

[8] H. Takai, [for the ATLAS Collaboration] Eur. Phys. J. C 34 (2004) S307.

[9] K. Adcox et al. [PHENIX Collaboration], Phys. Rev. Lett. 88 (2002) 022301.

[10] S. S. Adler et al. [PHENIX Collaboration], Phys. Rev. C 69 (2004) 034910.

[11] C. Adler et al. [STAR Collaboration], Phys. Rev. Lett. 89 (2002) 202301.

[12] J. Adams et al. [STAR Collaboration], Phys. Rev. Lett. 91 (2003) 172302.

[13] B. B. Back et al. [PHOBOS Collaboration], Phys. Lett. B 578 (2004) 297.

[14] I. Arsene et al. [BRAHMS Collaboration], Phys. Rev. Lett. 91 (2003) 072305.

[15] J. Adams et al. [STAR Collaboration], Phys. Rev. Lett. 93 (2004) 252301.

[16] C. Adler et al. [STAR Collaboration], Phys. Rev. Lett. 90 (2003) 082302.

[17] D. Magestro [STAR Collaboration], Nucl. Phys. A 774, 573 (2006).

[18] J. Adams et al. [STAR Collaboration], Phys. Rev. Lett. 97 (2006) 162301.

[19] X. N. Wang, Phys. Lett. B 579 (2004) 299.

[20] A. Dainese, C. Loizides and G. Paic, Eur. Phys. J. C 38 (2005) 461.

[21] K. J. Eskola, H. Honkanen, C. A. Salgado and U. A. Wiedemann, Nucl. Phys. A 747 (2005) 511.

[22] M. Gyulassy, I. Vitev and X. N. Wang, Phys. Rev. Lett. 86 (2001) 2537.

[23] T. Hirano and Y. Nara, Phys. Rev. C 66 (2002) 041901.

[24] T. Renk, J. Ruppert, C. Nonaka and S. A. Bass, Phys. Rev. C 75 (2007) 031902.

[25] C. A. Salgado and U. A. Wiedemann, Phys. Rev. Lett. 93 (2004) 042301.

[26] S. Pal and S. Pratt, Phys. Lett. B 574 (2003) 21.

[27] N. Borghini and U. A. Wiedemann, arXiv:hep-ph/0506218.

[28] A. Majumder, E. Wang and X. N. Wang, arXiv:nucl-th/0412061.

[29] N. Armesto, C. A. Salgado and U. A. Wiedemann, Phys. Rev. Lett. 93 (2004) 242301.

[30] I. Vitev, Phys. Lett. B 630 (2005) 78.

[31] A. D. Polosa and C. A. Salgado, Phys. Rev. C 75 (2007) 041901.

[32] I. P. Lokhtin, S. V. Petrushanko, L. I. Sarycheva and A. M. Snigirev, Phys. Atom. Nucl. 69 (2006) 1609 [Yad. Fiz. 69 (2006) 1643].

[33] M. Gyulassy and X. N. Wang, Nucl. Phys. B 420 (1994) 583.

[34] R. Baier, Y. L. Dokshitzer, A. H. Mueller, S. Peigne and D. Schiff, Nucl. Phys. B 484 (1997) 265.

[35] B. G. Zakharov, JETP Lett. 65 (1997) 615.

[36] U. A. Wiedemann, Nucl. Phys. B 588 (2000) 303.

[37] M. Gyulassy, P. Levai and I. Vitev, Nucl. Phys. B 594 (2001) 371.

[38] X. N. Wang and X. F. Guo, Nucl. Phys. A 696 (2001) 788.

[39] R. J. Fries, B. Muller, C. Nonaka and S. A. Bass, Phys. Rev. Lett. 90 (2003) 202303.

[40] D. Molnar and S. A. Voloshin, Phys. Rev. Lett. 91, 092301 (2003). 
[41] V. Greco, C. M. Ko and P. Levai, Phys. Rev. C 68 (2003) 034904.

[42] R. C. Hwa and C. B. Yang, Phys. Rev. C 67 (2003) 034902.

[43] R. J. Fries, B. Muller, C. Nonaka and S. A. Bass, Phys. Rev. C 68 (2003) 044902.

[44] L. Maiani, A. D. Polosa, V. Riquer and C. A. Salgado, Phys. Lett. B 645 (2007) 138.

[45] R. J. Fries and B. Muller, Eur. Phys. J. C 34 (2004) S279.

[46] E. Braaten and M. H. Thoma, Phys. Rev. D 44, 1298 (1991).

[47] M. Djordjevic, Phys. Rev. C 74 (2006) 064907.

[48] A. Adil, M. Gyulassy, W. A. Horowitz and S. Wicks, Phys. Rev. C 75 (2007) 044906.

[49] Yu. Dokshitzer and S. Troyan, XIX Winter School of LNPI (1984) Vol. 1, p. 144, preprint LNPI-922

[50] Y. L. Dokshitzer, V. A. Khoze and S. I. Troian, Adv. Ser. Direct. High Energy Phys. 5 (1988) 241.

[51] Y. L. Dokshitzer, V. A. Khoze and S. I. Troian, J. Phys. G 17 (1991) 1481.

[52] C. P. Fong and B. R. Webber, Nucl. Phys. B 355 (1991) 54.

[53] Y. I. Azimov, Y. L. Dokshitzer, V. A. Khoze and S. I. Troian, Z. Phys. C 27 (1985) 65.

[54] Y. I. Azimov, Y. L. Dokshitzer, V. A. Khoze and S. I. Troian, Z. Phys. C 31 (1986) 213.

[55] H. Aihara et al. [TPC/Two Gamma Collaboration], Phys. Rev. Lett. 52 (1984) 577.

[56] D. Acosta et al. [CDF Collaboration], Phys. Rev. D 68 (2003) 012003.

[57] D. K. Srivastava, C. Gale and R. J. Fries, Phys. Rev. C 67 (2003) 034903.

[58] R. Baier, Y. L. Dokshitzer, A. H. Mueller and D. Schiff, JHEP 0109 (2001) 033.

[59] B. A. Kniehl, G. Kramer and B. Potter, Nucl. Phys. B 582 (2000) 514.

[60] K. J. Eskola, H. Honkanen, H. Niemi, P. V. Ruuskanen and S. S. Rasanen, Phys. Rev. C 72 (2005) 044904 . 